\title{
Ophthalmic Examinations Link Identifier
}

National Cancer Institute

\section{Source}

National Cancer Institute. Ophthalmic Examinations Link Identifier. NCI Thesaurus. Code C162022.

A sequence of characters used as a linkage between related ophthalmic examinations identifier records. 